\title{
Community Generated Location Based Gaming
}

\author{
Kate Lund \\ Infolab21, Lancaster University \\ Lancaster, LA1 4WA, UK \\ m.lochrie@lancaster.ac.uk \\ k.lund1@lancaster.ac.uk
}

Paul Coulton p.coulton@lancaster.ac.uk

\begin{abstract}
This paper presents the concept for community driven Location Based Games (LBG) that involve actively exploring and discovering public spaces similar to the characteristics seen in Geocaching. The game demonstrated in this paper attempts to utilise the motivations and overcome the limitations of existing Location Based Games.
\end{abstract}

Location Based Games, User Generated Content, Emergent Behaviour, Mobile

\section{INTRODUCTION}

Recent research in this field has led us to the conclusion that there is potential opportunity for emergent Location Based Games that deviate away from players physical movement and encourages them to consider their location and its environment $[1,3]$.

The aim of this research seeks to generate a wider community that is specific and relevant to game play, with the aim to maintain and achieve longevity with an active following [8]. Some Location Based Services such as Geocaching ${ }^{1}$ have succeeded in making a significant impact upon the wider public reaching an estimated 3-4 million users worldwide [2, 8, http://www.geocaching.com]. This success can be attributable to the notion that Geocaching focuses on the players location and proximity rather than their physical action [7, 8] as seen in many other LBGs. The downsideto many types of LBGs is that they effectively remove one of the success factors seen in Geocaching, which is discovery and exploration of the specific location. LBGs are commonly independent of precise location, which is often deliberate as it makes games more scalable. This is why games like Foursquare [http://foursquare. com] or the Booyah Society [http://www.booyah. com] have become increasingly popularamongst players, as they try to connect the virtual game closer to real world location, through players capturing the locations they regularly visit as part of the game play. This capturing is then relayed to their 'friends' to generate social capital using established social networks such as Twitter and Facebook. Thus these games have a relatively simplistic game mechanic and their primary role would appear to serve as a means of informing their friends of their location which identifies them more closely to previous 'Friend Finder' services such as DodgeBall [4]. In this regard this type of gaming appears to be primarily aimed at young urban professionals with very active social lives that involve going out regularly. This inevitably means that such games offer little to families or groups who might use Geocaching as a means of engaging in highly social outdoor planned activities $[2,6,7]$. We attempt to draw on the positive attributes of these games and combine them to provide an allround positive experience that presents flexibility and encourages social interaction.

Furthermore, as there is little competitive element to Geocaching it lacks the required characteristics that are needed to categorise it as a game rather than a general activity [9]. We therefore believe there is an opportunity for creating a LBG that builds upon the motivations of Geocaching but accommodates competitive play. To this end we have created Big Game Huntr which can be regarded as a toolset for user generated location based games.

Big Game Huntr encourages emergent behaviour by promoting self-expressed design and creation of events [5], which can be shared within the community of registered players. The game premise requires participants to capture photographs of specified objects or actions to gain points and can be played either as a single group or amongst teams. The social capital generated within these games can also be fed into existing social networks such as Twitter and Facebook. Activities such as Geocaching rely on fixed pre-defined locations, whereby physical caches are already established in a position for players to locate. Big Game Huntr introduces the flexibility for players to design and create events in

\footnotetext{
${ }^{1}$ Geocaching is an outdoor activity where participants hunt for treasure using GPS technology. The games premise is to hide and seek hidden containers (also known as 'geocaches' or 'caches') of different sizes containing a logbook and occasionally travel toys, coins and souvenirs, players then shareyour experiences online amongst community members.
} 


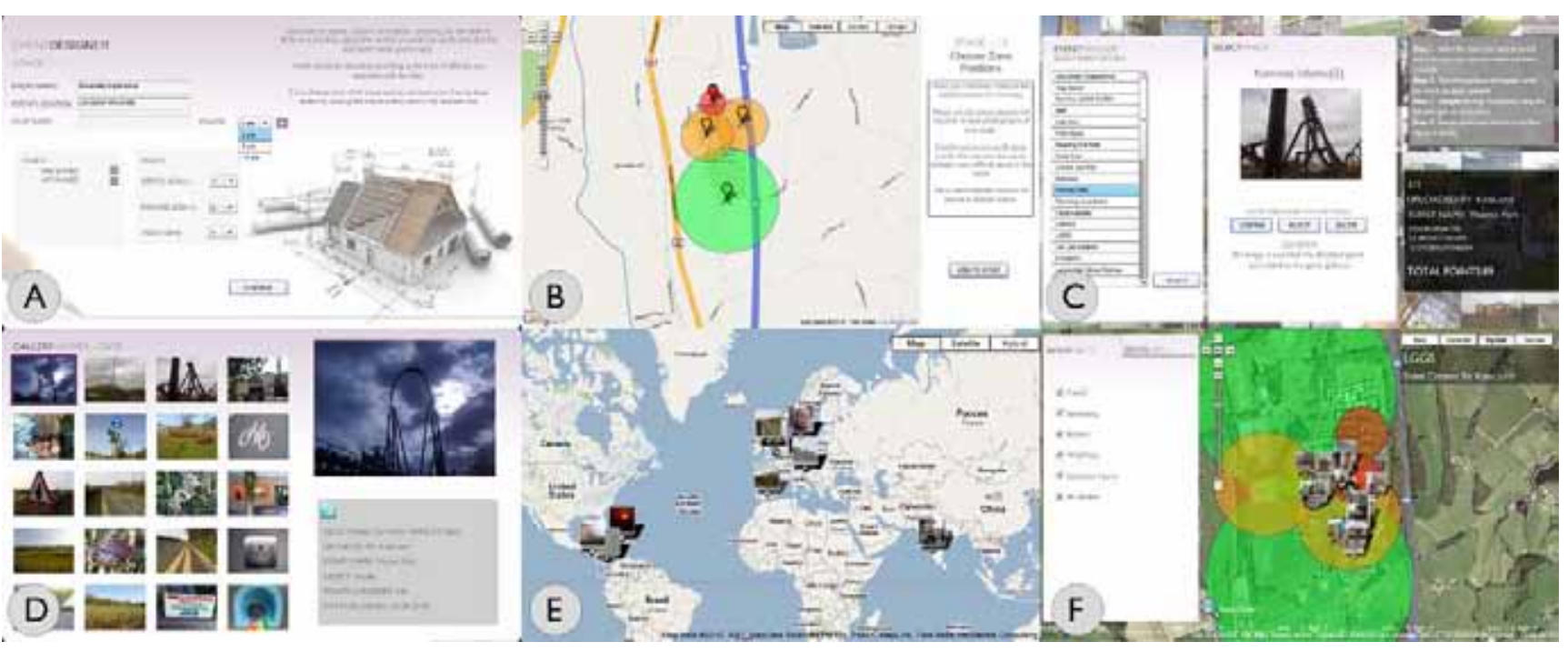

Figure 1. Screenshots of Big Game Huntr Web Design Portal taken from (www.biggamehuntr.com)

locations of their choosing remotely, without having to visit these areas to set up a game ahead of time. The game consists of two interfaces, an onli e web portal, available for players to design and create events, and a Flash Litemobile client used for accessing events created by the community and for playing the game.

\section{2. "BIG GAME HUNTR"}

\subsection{The Game}

Big Game Huntr is described by six distinct keywords; Design, Create, Play, Locate, Capture and Share, which summarise the main processes in the gameplay.

Players are given a large amount of freedom when designing games which allows them to be tailored to specific themes, locations, activities and objects with the aim of accommodating a wide demographic of potential gamers.

\subsubsection{Design}

Using the web based user design portal, players are given the freedom to create an event around their chosen topic. When creating an event, players need to consider their overall game arena (location and surrounding area), enforcing great emphasis on the actual location and its surroundings.

The game can be created at varying levels of difficulty, with points awarded which reflect this attribute. The target audience also needs to be taken into consideration, is this game to beplayed individually, or is it intended to have mass appeal and gain many participants.

\subsubsection{Create}

Once a user has registered and has a design concept for a game, they can set up their own event online using the tools of BigGameHuntr.com. To create an event players are required to set a list of clues (which can be objects, actions, emotions or descriptions) and a series of zones, strategically placed on a map which award players with different levels of points. Event creators are also responsible for maintaining their event, confirming that an image is of the correct object or action.

\subsubsection{Play}

Players can participate in their own created game, or alternatively can take part in events previously created by other members of the community. This depends largely upon whether the game is to fit a specific purpose (for example, to accompany a family outing), or if it is an activity carried out more spontaneously (for example, on the journey to work). Great emphasis is placed upon the strategy elements of game play. Players have to plan where they are going to go, which photos to capture, which zone and whereabouts within that zone to be in order to maximise the number of points awarded. Related to the strategic nature of the game, a competitive element is also provided via the leaderboards displayed throughout the Big Game Huntr website.

\subsubsection{Locate}

Players can choose from a list of created events, the player is required to travel to the appropriate zone(s) in which to capture a photo of the specified objects or actions. Players should be fully aware of their surroundings to capture the appropriate clue. This is particularly a benefit to the game as families, as an alternative method to engage their children with the environment they are visiting and attract attention to sights that they may normally overlook.

\subsubsection{Capture}

Once positioned within a zone, and satisfied with the location, the player is required to take a photo of one 
of the objects selected in the event, or perform the task that has been defined.

\subsubsection{Share}

All uploaded images are shared amongst the player community, which is generated through the website. The photos are shared in the form of galleries, and as markers on maps showing the location at which they were taken. Big Game Huntr has been designed to utilise existing social relationships to increase awareness of the game and incorporate user play into everyday life. All uploaded images are shared amongst the player community on the website, in the form of galleries, and as markers on maps showing the location at which they were taken. If the user registers with their Twitter details then the players game data and content is uploaded to their social network. It is also intended that the integration of social networks into the game will increase the awareness of the event that has been created, and publicise the game in general.

\section{2 "Big Game Huntr" Creation Interface}

As described, Big Game Huntr encourages players to express their creativity by allowing the players the flexibility to design and create their own event. Through the web based application players are given the freedom to create an event around their chosen topic.

In addition to the flexibilty the game design interface provides, the process offers further creative control, in respect that players need to consider their overall game arena (location and surrounding area), enforcing greater emphasis on the actual location. For example, players could create a zone of great difficulty for example on an island in a pond which could tax the ingenuity of those actually playing the games.

The game creation process requires the user to follow simple series of guided steps to achieve maximum flexibility in Big Game Huntr event design. Step 1: Define the name of the event along with a list of clues. The player must then select zone types (size and point value), and the location of the event. The size of the zone provides a degree of difficulty with smaller zones offering a greater number of points to players. As shown in Figure 1.A.

Step 2: Place zones around the specified locations. As illustrated in Figure 1.B which shows an event created at Lancaster University.

Once games are created and are being played the game creator can award points to photos uploaded from the mobile client. This is necessary as human validation is required to confirm that the uploaded photo matches the corresponding clue, as shown in Figure 1.C. Once photos have been uploaded and accepted they are browsable by other users in the community as shown in Figure1.D, 1.E and 1.F.
Players demonstrate they have completed a particular clue by taking a geo-located photograph as evidence. It is important to note that the objects or activities are not tied to a particular zone and the final score for completing a particular clue is a multiplication of the clue difficulty, distance from the centre of the zone and the zone difficulty. In this way players must carefully consider how to combine clues and zones to achieve the best score which turns the game from a run around and find game to a game reliant on strategy.

As photographs are subjective and the likeliness two players would produce the same result is very low, it is up to the game creator to accept the validity of the photograph and decide whether it warrants the number of points that have been generated (as shown in Figure 1.C). Photographs which aren't valid can still be accepted onto the system as user generated content but carry no effect on the competitive aspects of Big Game Huntr. The reason for this is to provide all users with a graphical representation of the world with all accepted photos plotted, showing the user where the game is being played in other locations (as shown inFigure 1.E). All uploaded photos can be viewed on the system through a range of visual representations such as galleries and plotted as markers on maps. Each photo that is plotted contains data from the user who uploaded the photo, when and how many points it was awarded. The users name appears in the info window if this user has a Twitter account then the name is represented in the form ofa link to their Twitter profile.

\section{3 "Big Game Huntr" Mobile Client}

Big Game Huntr's mobile counterpart is a Flash Lite mobile application developed initially for Nokia 5800 S60 5th edition touch screen phones utilising the most recent advances on the Flash Lite platform by extending its capabilities through the ApiBridge and Nokia's Platform Services.

The main operation for the mobile client is to provide the player with their current location, displayed graphically on the map (currently provided by Google) and to produce the evidence required in order to score points (as seen in Figure 2.B and 2.C).

Players must be registered before they can play the game which can either be done through the web portal or mobile client. In an attempt to create a viral awareness of the game it allows players to register using their Twitter ID which then allows them to publish their game data as tweets along with their photographs being uploaded to Twitpic.

The mobile client connects to the game server where it checks the users' authentication and then retrieves all active events from the database (as 
seen in Figure 2.A). Players then select a game event in which they wish to participate and the mobile client extracts thespecific game data (map, zones, and clues) from the server (as seen in Figure 2.B). Once this data is obtained the player can then start to play the game and the mobile client retrieves its current position coordinates using GPS (as seen in Figure 2.B). This position is shown on the game arena map (which is updated in real-time) along with the zones.

Players can reveal the list of clues to hunt at any time by swiping their finger across the screen (as seen in Figure 2.C, this is illustrated using the finger image representing a swiping motion on the screen from right to left). Accuracy and relevance within game play are enforced on players by restricting a player to one photo upload per clue (as seen in Figure 2.D). Once the photo is taken and uploaded the clue is removed from the list, meaning they cannot go back and take that clue again.

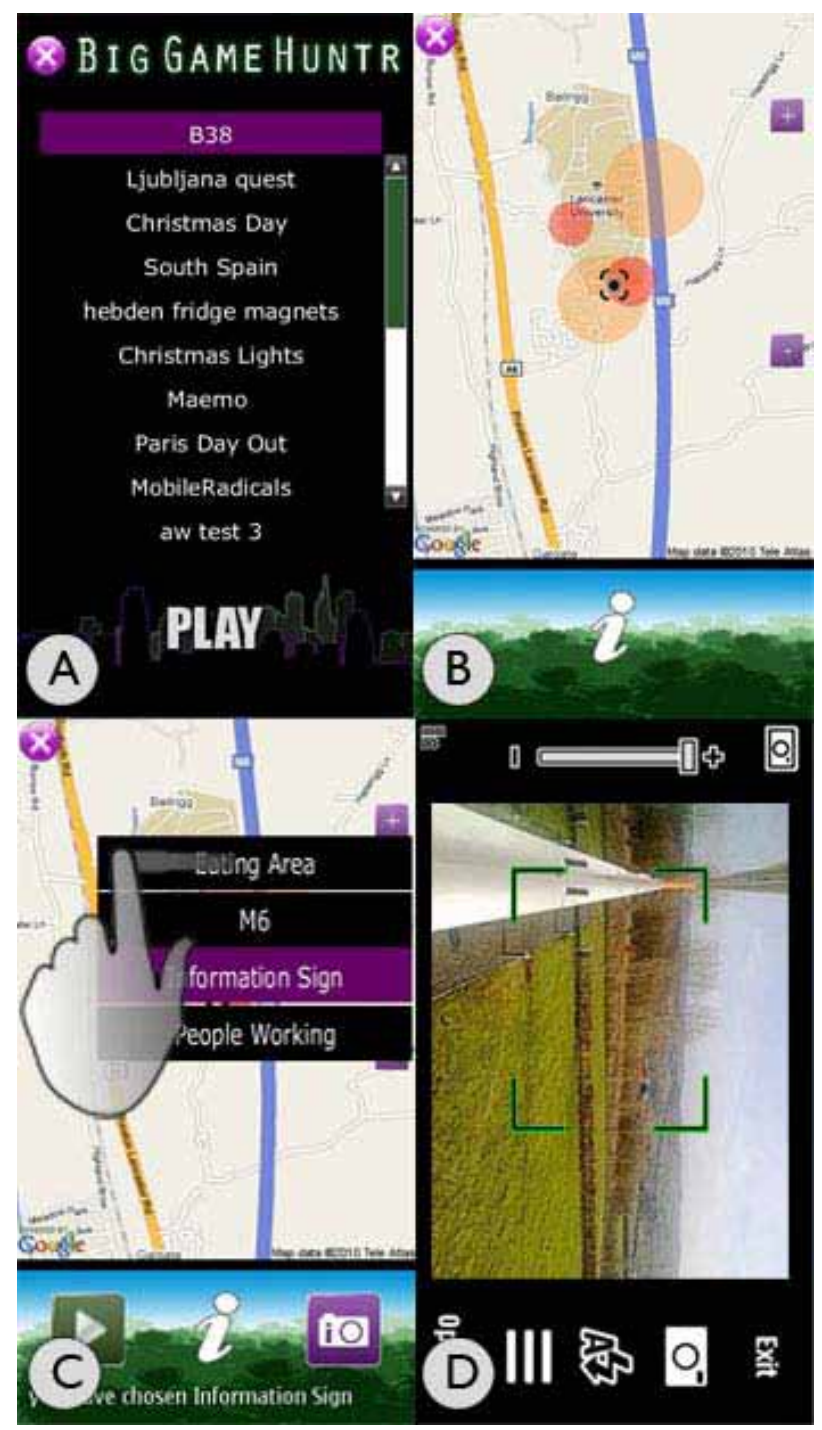

Figure 2. Screenshots of Big Game Huntr's Mobile Client using a Nokia N97.

\section{USER EXPERIENCE}

The game currently has 30 registered players ( 27 of which have registered using their twitter account), of which have created a total of 43 events, across 8 different countries. Between them they have uploaded (and verified) 290 photos. Thus far the game has been running for four months in a beta prerelease. This period has given us the opportunity to collect statistics based upon player interactions and demonstrates the potential extent of usergenerated content, both in the creation of events and in the participation of uploading photographs.

So far the types of events and clues that users have created range from landmarks (to seek) to more creative interpretations of the games capabilities such as ("Christmas Lights" - based on the quality of house decorations) to actions (performing an action such as the conga).

Big Game Huntr offers multiple methods for participation, the game could be applied to a social situation, on a small scale; a family could use the game to pass the time taking exercise while walking in the countryside, getting family members to participate in thinking what they are going to see and where they might see these things [5, 7]; on a larger scale the game could be applied to festivals and other large organised events, such as Glastonbury, to provide visitors with interactive content.

The game helps the user to identify landmarks and makes them more aware of their immediate surroundings, as they are actively looking for the clue. This factor links into the idea of the desire to explore and discover [7] the opportunities of seeing new places which without the motivation of playing the game may have remained unseen.

Finally the competitive aspect of the games point scoring and leader board, could have strong motivational appeal, as people aim to see their name in the list of top scoring players, or attempt to gain a higher score than their friends.

There are also motivational factors in the creation of an event. Firstly this allows the user to create an event tailored to somethingspecific from their perspective; they can create an event with a specific audience in mind, or to accompany another event, such as an excursion. A certain amount of satisfaction can also be gained by seeing other players participate in your game which you designed, and being able to generate images from a wider range of sources thus increasing the amount of images around your chosen subject.

\section{CONCLUSION}

The project presented in this paper is research in progress. Big Game Huntr addresses the issues surrounding LBG's by seeking to incorporate user creativity, exploration and discovery and thus promote 
emergent behaviour in a flexible environment in order to provide an outlet for generated content, driven by communities.

Once the game is release in to the public domain our long term aims are to implement the foundations set by Big Game Huntr as a platform for the study of longitudinal practices of these types of games. Focussing on LBG's for social play thus far has not been addressed in previous research and will provide general insights applicable to future Location Based Services.

\section{ACKNOWLEDGMENTS}

The authors wish to acknowledge Nokia support for providing the mobile devices used in the development of "Big Game Huntr".

[1] Benford, S., Magerkurth, C., and Ljungstrand, P. Bridging the physical and digital in pervasive gaming. Commun. ACM 48, 3 (Mar. 2005), pp.54-57. [2] Chavez, D.J., Schneider, I., and Powell, T. The social-psychology of a technology driven outdoor trend: Geocaching in the USA. Hawaii International Conference on Social Sciences, 2004 June 16-19, Honolulu, pp. 583-594.

[3] Coulton, P., Lund, K., Wilson, A. Harnessing Player Creativity to Broaden the Appeal of Location Based Games. Accepted for $\mathrm{HCl}$ 2010: Play is a serious business, 6-10 Sept 2010.

[4] Humphreys, L. . Mobile social networks and social practice: A case study of Dodgeball. Journal of Computer-Mediated Communication, 13(1), article 17, 2007.

[5] Jegers, K. Wiberg, M. Pervasive Gaming in the Everyday World, IEEE Pervasive Computing, vol. 5, no. 1, pp. 78-85, Jan.-Mar. 2006.

[6] Kelly, M. Local Treasures: Geocaching across America, Center for American Places, February 15, 2006

[7] O'Hara, K. Understanding Geocaching Practices and Motivations. Proceeding of the twenty-sixth annual SIGCHI conference on Human factors in computing systems. Florence, Italy, 2008, pp.11771186.

[8] Rashid, O., Mullins, I., Coulton, P., and Edwards, R. Extending cyberspace: location based games using cellular phones. Comput. Entertain. 4, 1 (Jan. 2006), 4.

[9] Salen, K and Zimmerman, E. Rules of Play: Game Design Fundamentals, The MIT Press, October 2003. 